\title{
Power deposition outside the divertor in ASDEX Upgrade
}

\author{
A. Herrmann, T. Eich, V. Rohde, C.J. Fuchs, J. Neuhauser, ASDEX Upgrade team \\ Max-Planck-Institut für Plasmaphysik, EURATOM-IPP Association, \\ Garching und Greifswald, Germany
}

\section{Introduction}

The heat flux to the lower (main) divertor in ASDEX Upgrade is routinely monitored with a high resolution IR line camera. A 2D IR camera system measuring the heat load to the upper divertor came into operation recently. Radiative power losses from the plasma, in the scrape-off layer, and the divertor are measured by different bolometer systems. These measurements are used to establish ELM averaged power balances for $\mathrm{H}$-mode discharges which are balanced inside error bars of $\pm 20 \%$ [1]. Investigation of ELM energy losses at ASDEX Upgrade and JET revealed that the energy detected in the divertor accounts for only about $50 \%$ of the midplane losses [2]. The missing part could be dissipated by different mechanisms. (i) A strong increase of the radiation level in the scrape off layer and the divertor region or (ii) by rapid radial diffusion or even outward drift [3]. (i) would require to dissipate up to $5 \%$ of the stored plasma energy by radiation. The required radiation level exceeds the averaged radiation level by one order of magnitude. This is investigated experimentally with bolometer measurements of adequate time resolution and time resolved divertor thermography. (ii) ELM energy losses by rapid radial transport and heat deposition outside the divertor, keeping the heat deposition profile in the divertor during an ELM similar to the inter ELM profile, should be detectable as surface temperature increase of in vessel components, if such mechanism of energy transport contributes significantly to the over all ELM energy balance. The heat deposition to non divertor in vessel components was experimentally investigated in ASDEX Upgrade and the experimental results are presented in this paper.

\section{Diagnostics and Experiments}

The main diagnostic tool for the measurement of in vessel heat load is a $2 \mathrm{D}$ IR camera system, working in snapshot mode down to $4 \mu$ integration time at frame rates of 315 full frames/s (320x256 pixels) or $10^{4}$ frames/s with reduced frame size $(128 \times 8$ pixels $)$. The camera is mounted in the midplane of ASDEX Upgrade viewing tangential into the vessel (Fig. 1). Essentially all components expected to receive energy (inner heat shield, ICRH antenna, and via a mirror, the protection limiter) are in the field of view of the system. The snapshot mode allows to detect the expected events, indeed with aliasing effects. From the measured temperature evolution the heat flux is calculated using the 1D option of the 2D Theodor code [4]. Thermocouples inside limiter and inner column carbon tiles are used to measure the total energy

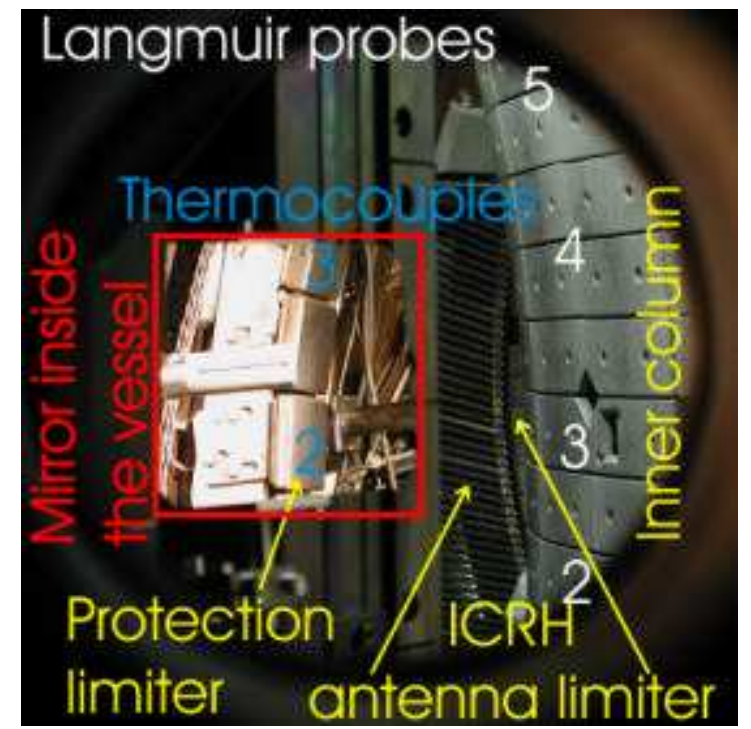

Fig. 1: Midplane view into the ASDEX Upgarde from the position of the IR camera.

deposited during a single shot. In addition the particle flux to the central column is measured by Langmuir probe tips swept with a time resolution of $1 \mathrm{~ms}$ per characteristic.

The measurements presented in this paper were performed in standard $\mathrm{H}$-mode discharges with magnetic lower single single null configuration. The heating power was stepwise increased from 2.5 to $5 \mathrm{MW}$ establishing a stable $\mathrm{H}$-mode with type I ELMs, which are expected to be the most significant events for heat deposition during regular operation in ITER. The plasma current was 
$I_{P}=1 \mathrm{MA}$, and the magnetic field, $B_{t}=-2.0 \mathrm{~T}$, resulting in an ion-grad $B$ drift direction towards the lower active divertor.

\section{Results and Discussion}
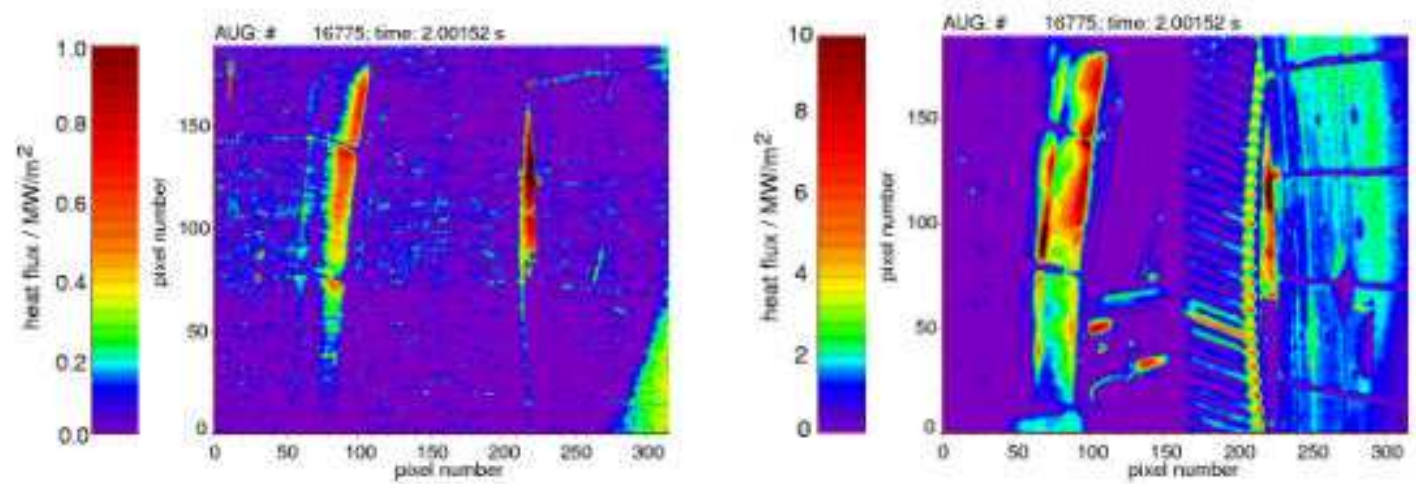

Fig. 2: Calculated heat flux to in vessel components of ASDEX Upgrade in between ELMs (left) and for a typical ELM (right). In between ELMs the protection limiters are loaded only, whereas during an ELM also a part of the inner column receives up to $2 \mathrm{MW} / \mathrm{m}^{2}$.

Calculated heat flux patterns in between ELMs (left) and during a typical ELM (right) are shown in Fig. 2. In between ELMs only the protection and the ICRH antenna limiters are significantly loaded at the leading edge with up to $1 \mathrm{MW} / \mathrm{m}^{2}$. The heat flux to the central column is below the detection limit of $100 \mathrm{~kW} / \mathrm{m}^{2}$. During an ELM the heat flux increases by about a factor of 10 . The heat flux at the limiter exceeds $10 \mathrm{MW} / \mathrm{m}^{2}$ and parts of the inner column are loaded with up to $2 \mathrm{MW} / \mathrm{m}^{2}$. The inclination of the limiter tiles with respect to the magnetic field lines is about 7 degrees so that the parallel heat flux in front of the limiter is about $8 \mathrm{MW} / \mathrm{m}^{2}$ in between ELMs and up to $80 \mathrm{MW} / \mathrm{m}^{2}$ during an ELM. These values are comparable to the heat flux measured with thermography in the lower divertor far away from the strike point position as shown in Fig. 3. The flat tail of the divertor heat flux profile extends up to the limiter position about $3 \mathrm{~cm}$ away from the outermost divertor measurement. This is consistent with the SOL structure described by steep gradients of $T_{e}$ and $n_{e}$ across the separatrix linked to each other by the condition $\eta_{e}=2$ and flat profiles in regions far away from the separatrix [3].

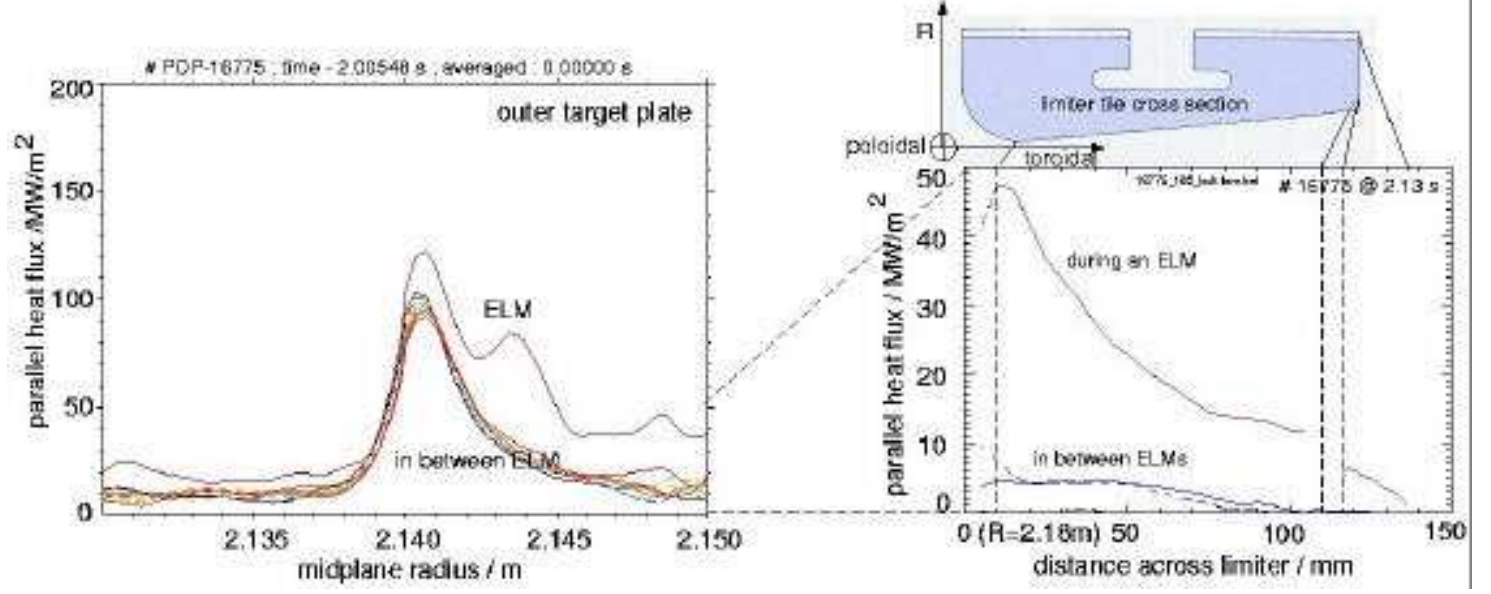

Fig. 3: Parallel heat flux in the lower outer divertor (left) and at a limiter tile of the protection limiter. The heat flux at the leading edge of the limiter is comparable with the heat flux observed at the outer part of the lower divertor. The parallel heat flux at the end of the inclined limiter part and the nearly perpendicular part is comparable, i.e. the 'second' peak at the limiter tiles in Fig. 2 is due to the high pitch angle at the perpendicular part of the limiter.

At the inclined part of the limiter, there is a clear radial decay of the deposited heat flux with an efolding length of about $50 \mathrm{~mm}$ along the limiter, and $7 \mathrm{~mm}$ along major radius, respectively. In 
addition, Fig. 3 shows a nearly continuos parallel heat flux profile also in the region where the inclination of the limiter surface compared to the magnetic field lines changes, i.e. the 'second' peak at the limiter tiles in Fig. 3 is due to the higher pitch angle at the perpendicular part of the limiter and not a discontinuity in heat deposition. The energy deposited during an ELM to the upper limiter tile in the field of view of the IR camera is $75 \mathrm{~kW} \times 0.75 \mathrm{~ms}=56 \mathrm{~J}$.

The majority of the observed ELMs shows a smooth heat flux variation along the imaged limiter section, similar to the ELM shown in Fig. 2, with some modulation on shorter scales. Only 2 out of 30 ELM frames analysed show narrow events on a few $\mathrm{cm}$ scale (Fig.4). One should notice that, for field aligned modes, a full poloidal wavelength of $25 \mathrm{~cm}$ (about the mirror height) corresponds to a toroidal wavenumber $\mathrm{n} \sim 11$. Shorter scales indicate admixtures with higher $\mathrm{n}$, while modes with lower wavenumbers are increasingly difficult to identify in the present set-up. This result is consistent with the quasi-mode spectrum derived from the upper divertor heat flux pattern using the same camera $[5$, 6], and also with findings from on JET [7].
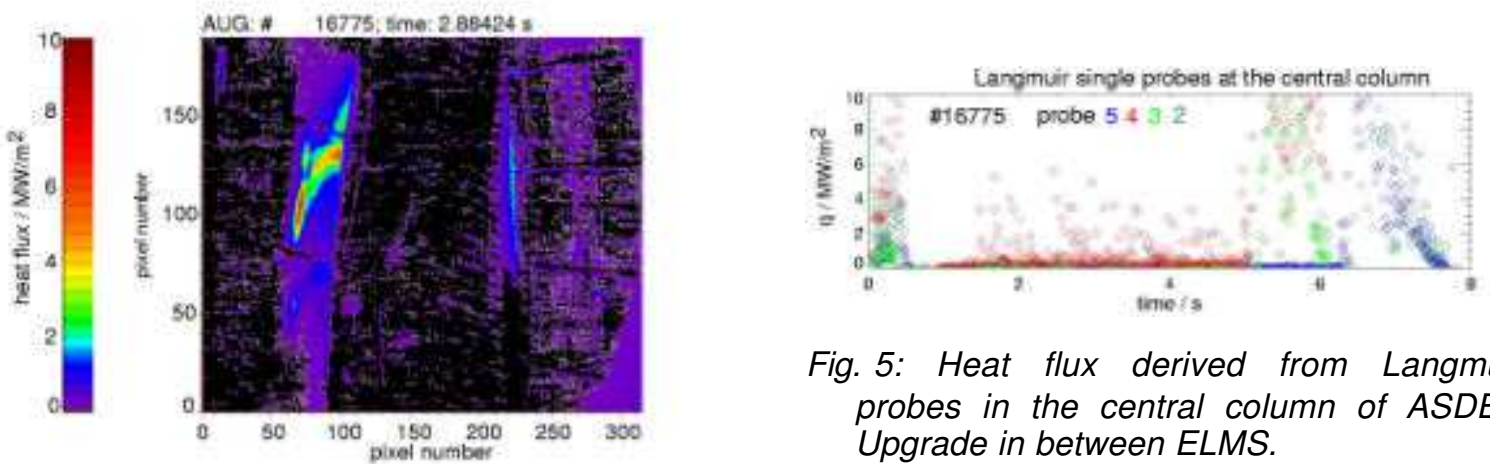

Fig. 5: Heat flux derived from Langmuir probes in the central column of ASDEX Upgrade in between ELMS.

Fig. 4: Snapshot of an early ELM phase with a structured heat deposition at the limiter.

Langmuir probes are used to measure particle flux and energy to the inner column at one toroidal and 4 poloidal positions (the poloidal positions are indicated in Fig. 1. The probes are not in the field of view of the IR camera due to the toroidal position 160 degrees away from the indication). The resulting heat flux $\left(\gamma=7, E_{\text {ion }}=13.6 \mathrm{eV}\right)$ is shown in Fig. 5 .

A significant heat load of up to $10 \mathrm{MW} / \mathrm{m}^{2}$ to the central column is observed during the start up and ramp down phase only. The heat flux during the flat top phase of the discharge is significantly lower with an averaged value below $200 \mathrm{~kW} / \mathrm{m}^{2}$ in correspondence with thermographic measurements. The heat deposition during a single ELM is not resolvable due to the time necessary to record a Langmuir characteristics. Therefore data points from different ELMs are used to construct averaged ELM characteristics [8] for time values near to the ELM with the $H_{\alpha}$ signal as ELM marker. The resulting temporal evolution of electron temperature and density show a clear separation of the temperature and density maximum as shown in Fig. 6. Thereby, the density increase is partly due to enhanced recycling in the after ELM phase [9]. The resulting heat flux to the inner column is below $1 \mathrm{MW} / \mathrm{m}^{2}$ during an ELM and in the after ELM phase with increased density at moderate temperature, respectively.

coherent averaged ELM - Central column Probe 3
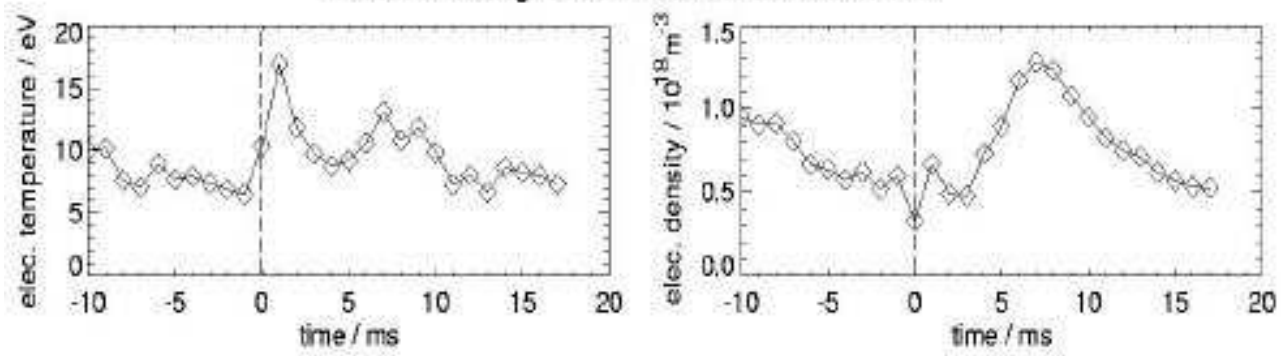

Fig. 6: Temporal evolution of electron temperature and -density for an coherent averaged ELM. The maximum of density and temperature are separated. 


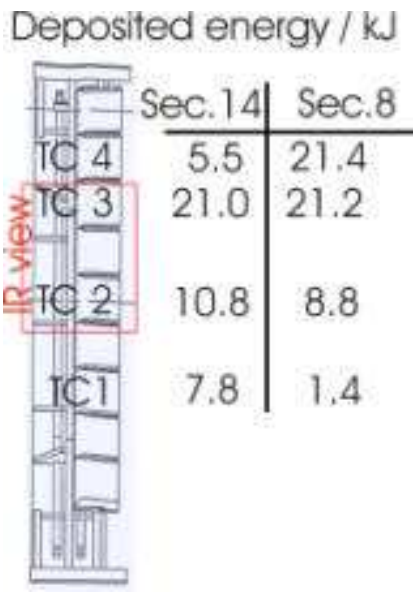

Fig. 7: Energy to carbon tiles of the Protection limiters in ASDEX Upgrade as measured with thermometry. The position of the Thermocouples and the field of view of the IR camera is indicated.
The IR temperature measurement is limited to 4 tiles of one limiter and one section of the central column. Therefore thermometry is used to get information about the poloidal and toroidal distribution of the energy load as summarised in Fig. 7 for the energy deposition to the protection limiters. The toroidal variation of the deposited energy to the main parts of the limiters (TC 3,2) is in the order of $10 \%$. The total energy received by 4 protection limiters becomes in total $460 \mathrm{~kJ}$ with the following assumption for the peaking factors: the top 4 tiles receives $20 \mathrm{~kJ}$ each, the next 2 tiles half, and the bottom 3 tiles a quarter of the energy to the top tiles $\left(\left(4^{\star} 20+2^{*} 10+3^{\star} 5\right)^{\star} 4 \mathrm{~kJ}\right)$. Together with the energy to the ICRH antenna limiters of $990 \mathrm{~kJ}$ from cooling water calorimetry the total load to the limiter is about $1.5 \mathrm{MJ}$. The heat load to the inner column is about $800 \mathrm{~kJ}$, with an area of about $7.5 \mathrm{~m}^{2}$. The total plasma input energy is $31.5 \mathrm{MJ}$, i.e. the energy to the limiters is below $5 \%$ of the input energy and about $8 \%$ of the energy to the lower divertor.

Using the same peaking factors as for the energy distribution, the ELM energy load to all limiters from thermography becomes: $56 \mathrm{~J} / E L M * 4 *\left(1_{\text {protection }}+2_{\text {ICRH }}\right) *(4+2 / 2+3 / 4)=3.8 \mathrm{~kJ} / E L M$. The

plasma energy loss during an ELM is $25 \mathrm{~kJ}$. Together with the ELM energy to the central column of $2.6 \mathrm{~kJ}\left(1 \mathrm{MW} / \mathrm{m}^{2 *} 3.5 \mathrm{~m}^{2 *} 0.75 \mathrm{~ms}\right)$ the ELM deposited energy to the limiters and the central column is $25 \%$.

\section{Summary and Conclusions}

A combination of diagnostics with high time resolution, such as thermography and Langmuir probes in combination with slow but toroidally and poloidally distributed measurements, such as thermometry, and cooling water calorimetry was used to estimate the heat deposition outside the divertor region in ASDEX Upgrade. The total deposited energy to non divertor components is below $10 \%$ of the plasma deposited energy. The main part of these energy is deposited onto the ICRH antenna limiters. The fraction of energy received by the central column during the flat top phase is negligible. The radial heat flux deposition profile is the continuation of the heat flux profile measured in the lower divertor. 2 of 30 ELM snapshots show a heat flux pattern different from the averaged one. The ELM contribution to the limiter load is in the order of $20 \%$. The fraction of ELM midplane loss received outside the divertor is about 0.25 .

\section{References}

1. J.C. Fuchs, D. Coster, A. Herrmann et al., J. Nucl. Mater., 290-293, 525 (2001).

2. A. Herrmann, T. Eich, S. Jachmich et al., J. Nucl. Mater., 313-316, 759 (2003).

3. J. Neuhauser, D. Coster, H.U. Fahrbach et al., Plasma Phys. Controlled Fusion, 44, 855 (2002).

4. A. Herrmann, W. Junker, K. Günther et al., Plasma Phys. Controlled Fusion, 37, 17 (1995).

5. T. Eich, A. Herrmann, and J. Neuhauser, This conference, (2003).

6. T. Eich, A. Herrmann, J. Neuhauser et al., Phys Rev. Letters, submitted (2003).

7. P. Ghendrih, Y. Sarazin, M. Becoulet et al., Journal of Nuclear Materials, 313, 914 (2003).

8. M. Laux, S. Jachmich, A. Herrmann et al., Europhysics Conference Abstracts (CD-ROM, Proc. of the 28th EPS Conference on Controlled Fusion and Plasma Physics, Madeira 2001), p. 1625 (2001).

9. $\quad$ G. Haas, R. Maingi, J. Neuhauser et al., J. Nucl. Mater., 266-269, 1065 (1999). 